\title{
Cross section for charmonium absorption by nucleons
}

\author{
W. Liu, C. M. Ko, and Z. W. Lin \\ Cyclotron Institute and Physics Department, Texas A\&M University, College Station, Texas 77843-3366
}

(Received 20 July 2001; published 3 December 2001)

\begin{abstract}
The cross section for $J / \psi$ absorption by nucleons is studied using a gauged SU(4) hadronic Lagrangian but with empirical particle masses, which has been used previously to study the cross sections for $J / \psi$ absorption by pion and $\rho$ meson. Including both two-body and three-body final states, we find that with a cutoff parameter of $1 \mathrm{GeV}$ at interaction vertices involving charm hadrons, the $J / \psi-N$ absorption cross section is at most $5 \mathrm{mb}$ and is consistent with that extracted from $J / \psi$ production from both photonuclear and proton-nucleus reactions.
\end{abstract}

DOI: 10.1103/PhysRevC.65.015203

PACS number(s): 14.20.Dh, 13.75.Lb, 14.40.Lb, 25.75.-q

\section{INTRODUCTION}

Two main mechanisms for $J / \psi$ suppression observed in relativistic heavy ion collisions [1] are the dissociation by the quark-gluon plasma [2] and the absorption by comoving hadrons, mainly, pions and $\rho$ mesons [3]. The cross sections for $J / \psi$ absorption by hadrons are, unfortunately, not well determined. In the perturbative QCD approach [4], based on the dissociation of charmonium bound states by energetic gluons inside hadrons, the dissociation cross section increases monotonously with the kinetic energy of hadrons and has a value of only about $0.1 \mathrm{mb}$ at $0.8 \mathrm{GeV}$. On the other hand, both the quark-interchange model [5] based on gluonexchange potentials and the meson-exchange model [6-8] based on hadronic Lagrangians give the absorption cross sections of $J / \psi$ by pion and $\rho$ meson that are more than an order of magnitude larger, i.e., a few millibarn. A similar magnitude for the $J / \psi-\pi$ absorption cross section has also been obtained in the QCD sum rules [9].

Since the absorption cross sections of $J / \psi$ by pion and $\rho$ meson cannot be directly measured, it is useful to find empirical information that can constrain their values. One such constraint is the cross section for $J / \psi$ absorption by a nucleon, as this process can be viewed as $J / \psi$ absorption by the virtual pion and $\rho$ meson from the nucleon. From $J / \psi$ production in photonucleus reactions, the cross section for $J / \psi$ absorption by nucleons can be extracted, and its magnitude has been found to be about $4 \mathrm{mb}$ [10]. The $J / \psi-N$ absorption cross section has also been extracted from protonnucleus collisions at proton energies from 200 to $800 \mathrm{GeV}$, and the empirical value is about $7 \mathrm{mb}$ [11].

In the meson-exchange model of Refs. [6-8], the interaction Lagrangians between pseudoscalar and vector mesons are obtained from the SU(4) invariant free Lagrangian for pseudoscalar mesons by treating vector mesons as gauge particles. This then leads to not only pseudoscalar-pseudoscalar vector-meson couplings but also three-vector-meson and four-point couplings. Since the SU(4) symmetry is explicitly broken by hadron masses, empirical hadron masses are used in the Lagrangian. Furthermore, values for the coupling constants are taken either from empirical information if they are available or from theoretical models, such as the vectormeson dominance model and the QCD sum rules. Otherwise, they are determined by using relations derived from the SU(4) symmetry. In this paper, we shall generalize this La- grangian to study the absorption cross section of $J / \psi$ by nucleons and to see if its magnitude is consistent with that extracted from $J / \psi$ production in photonucleus and protonnucleus reactions.

This paper is organized as follows. In Sec. II, we first consider $J / \psi$ absorption by nucleons via pion and $\rho$ meson exchange. The process of $J / \psi$ absorption by nucleons via charm exchange is studied in Sec. III. The effect due to the anomalous parity interaction of $J / \psi$ with charm mesons is studied in Sec. IV. In Sec. V, the total cross section for $\mathrm{J} / \psi$ absorption by nucleons is given. Finally, conclusions and discussions are given in Sec. VI. An Appendix is included to derive the SU(4) relations for some of the coupling constants involving charm hadrons.

\section{II. $J / \psi$ ABSORPTION BY NUCLEONS VIA PION AND $\rho$ MESON EXCHANGE}

Possible processes for $J / \psi$ absorption by nucleons involving virtual pion and $\rho$ meson are $J / \psi N$ $\rightarrow D^{*} \bar{D} N\left(\bar{D}^{*} D N\right), J / \psi N \rightarrow D \bar{D} N$, and $J / \psi N \rightarrow D^{*} \bar{D}^{*} N$, as shown by the diagrams in Fig. 1. The cross sections for these processes can be evaluated using the Lagrangians introduced in [6-8] for $J / \psi$ absorption by real pion and $\rho$ meson and in Ref. [12] for charm meson scattering by these hadrons.

The interaction Lagrangian densities that are relevant to the present study are given as follows:

$$
\begin{aligned}
& \mathcal{L}_{\pi N N}=-i g_{\pi N N} \bar{N} \gamma_{5} \vec{\tau} N \cdot \vec{\pi} \\
& \mathcal{L}_{\rho N N}=g_{\rho N N} \bar{N}\left(\gamma^{\mu} \vec{\tau} \cdot \vec{\rho}_{\mu}+\frac{\kappa_{\rho}}{2 m_{N}} \sigma^{\mu \nu} \vec{\tau} \cdot \partial_{\mu} \vec{\rho}_{\nu}\right) N \\
& \mathcal{L}_{\pi D D *}=i g_{\pi D D *} D^{* \mu} \vec{\tau} \cdot\left(\bar{D} \partial_{\mu} \vec{\pi}-\partial_{\mu} \bar{D} \vec{\pi}\right)+\text { H.c. } \\
& \mathcal{L}_{\rho D D}=i g_{\rho D D}\left(D \vec{\tau} \partial_{\mu} \bar{D}-\partial_{\mu} D \vec{\tau} \bar{D}\right) \cdot \vec{\rho}^{\mu}
\end{aligned}
$$

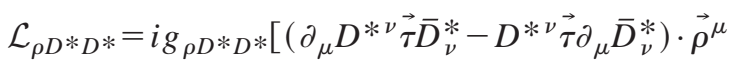

$$
\begin{aligned}
& +\left(D^{* \nu} \vec{\tau} \cdot \partial_{\mu} \vec{\rho}^{\nu}-\partial_{\mu} D^{* \nu} \vec{\tau} \cdot \vec{\rho}_{\nu}\right) \bar{D}^{* \mu} \\
& \left.+D^{* \mu}\left(\vec{\tau} \cdot \vec{\rho}^{\nu} \partial_{\mu} \bar{D}_{\nu}^{*}-\vec{\tau} \cdot \partial_{\mu} \vec{\rho}^{\nu} \bar{D}_{\nu}^{*}\right)\right]
\end{aligned}
$$






(1a)

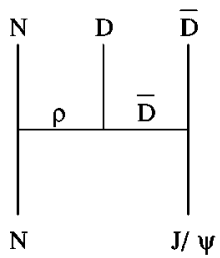

(2a)

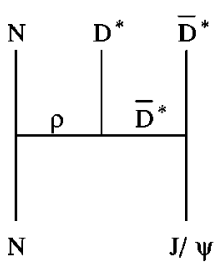

(3a)

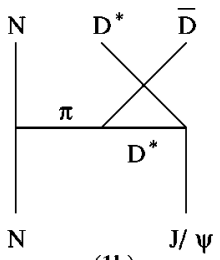

(1b)

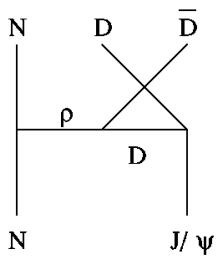

(2b)

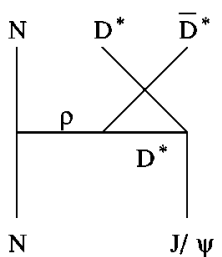

(3b)

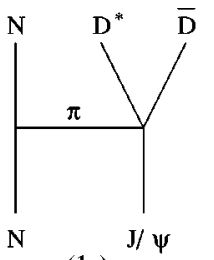

(1c)

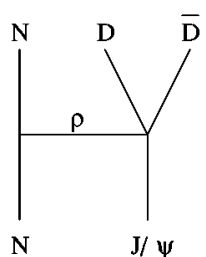

(2c)

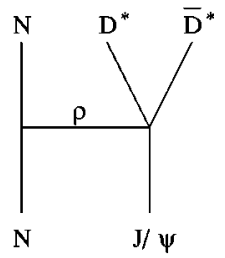

(3c)
FIG. 1. $J / \Psi$ absorption by nucleon via pion and $\rho$ meson exchanges.

$$
\begin{aligned}
& \mathcal{L}_{\psi D D}=i g_{\psi D D} \psi^{\mu}\left[D\left(\partial_{\mu} \bar{D}\right)-\left(\partial_{\mu} D\right) \bar{D}\right], \\
& \mathcal{L}_{\psi D^{*} D^{*}}=i g_{\psi D^{*} D^{*}[}\left[\psi^{\mu}\left(\partial_{\mu} D^{* \nu} \bar{D}_{\nu}^{*}-D^{* \nu} \partial_{\mu} \bar{D}_{\nu}^{*}\right)+\left(\partial_{\mu} \psi^{\nu} D_{\nu}^{*}\right.\right. \\
& \left.-\psi^{\nu} \partial_{\mu} D_{\nu}^{*}\right) \bar{D}^{* \mu}+D^{* \mu}\left(\psi^{\nu} \partial_{\mu} \bar{D}_{\nu}^{*}-\partial_{\mu} \psi^{\nu} \bar{D}_{\nu}^{*}\right), \\
& \mathcal{L}_{\pi \psi D D^{*}}=-g_{\pi \psi D D} \psi^{\mu}\left(D_{\mu}^{*} \vec{\tau} \bar{D}+D \vec{\tau} \bar{D}_{\mu}^{*}\right) \cdot \vec{\pi}, \\
& \mathcal{L}_{\rho \psi D D}=g_{\rho \psi D D} \psi^{\mu} D \vec{\tau} \bar{D} \cdot \vec{\rho}_{\mu}, \\
& \mathcal{L}_{\rho \psi D^{*} D^{*}}=g_{\rho \Psi D^{*} D^{*}}\left(\psi^{\nu} D_{\nu}^{*} \vec{\tau} \bar{D}_{\mu}^{*}+\psi^{\nu} D_{\mu}^{*} \vec{\tau} \bar{D}_{\nu}^{*}\right. \\
& \left.-2 \psi_{\mu} D^{* \nu} \vec{\tau} \bar{D}_{\nu}^{*}\right) \cdot \vec{\rho}^{\mu}
\end{aligned}
$$

In the above, $\vec{\tau}$ are Pauli spin matrices, and $\pi$ and $\rho$, denote the pion and $\rho$ meson isospin triplet, respectively, while $D=\left(D^{0}, D^{+}\right)$and $D^{*}=\left(D^{* 0}, D^{*+}\right)$ denote the pseudoscalar and vector charm meson doublets, respectively. The $J / \psi$ is denoted by $\psi$ while $N$ represents the nucleon.

For coupling constants, we use the empirical values $g_{\pi N N}=13.5[13], g_{\rho N N}=3.25, \kappa_{\rho}=6.1 \quad[14]$, and $g_{\pi D D *}$ $=4.4$ [15]. From the vector dominance model, we have $g_{\rho D D}=g_{\rho D * D *}=2.52$ and $g_{\psi D D}=g_{\psi D * D *}=7.64$ [7]. For the four-point coupling constants, we relate their values to the three-point coupling constants using the SU(4) relations [7], i.e.,

$$
\begin{gathered}
g_{\pi \psi D D}=g_{\pi D D} g_{\psi D D}, \quad g_{\rho \psi D D}=2 g_{\rho D D} g_{\psi D D}, \\
g_{\rho \psi D} * D * \\
=g_{\rho D} * D * g_{\psi D} * D * .
\end{gathered}
$$

The amplitudes for the first two processes in Fig. 1 are given by

$$
M_{1}=-i g_{\pi N N} \bar{N}\left(p_{3}\right) \gamma_{5} N\left(p_{1}\right) \frac{1}{t-m_{\pi}^{2}}\left(M_{1 a}+M_{1 b}+M_{1 c}\right),
$$

$$
\begin{aligned}
M_{2}= & g_{\rho N N} \bar{N}\left(p_{3}\right)\left[\gamma^{\mu}+i \frac{\kappa_{\rho}}{2 m_{N}} \sigma^{\alpha \mu}\left(p_{1}-p_{3}\right)_{\alpha}\right] \\
& \times N\left(p_{1}\right)\left[-g_{\mu \nu}+\frac{\left(p_{1}-p_{3}\right)_{\mu}\left(p_{1}-p_{3}\right)_{\nu}}{m_{\rho}^{2}}\right] \frac{1}{t-m_{\rho}^{2}} \\
& \times\left(M_{2 a}^{\nu}+M_{2 b}^{\nu}+M_{2 c}^{\nu}\right),
\end{aligned}
$$

where $p_{1}$ and $p_{3}$ are the four momenta of the initial and final nucleons, respectively. In the above, $M_{1 a}, M_{1 b}, M_{1 c}$ are the amplitudes for the subprocess $J / \psi \pi \rightarrow D^{*} \bar{D}$ in the top three diagrams of Fig. 1, while $M_{2 a}^{\nu}, M_{2 b}^{\nu}, M_{2 c}^{\nu}$ are the amplitudes for the subprocess $J / \psi \rho \rightarrow D \bar{D}$ in the middle three diagrams. The amplitude for the third process in Fig. 1 has a similar expression as that for the second process with $M_{2 a}^{\nu}, M_{2 b}^{\nu}, M_{2 c}^{\nu}$ replaced by $M_{3 a}^{\nu}, M_{3 b}^{\nu}, M_{3 c}^{\nu}$, which are the amplitudes for the subprocess $J / \Psi \rho \rightarrow D^{*} \bar{D}^{*}$ in the bottom three diagrams. Expressions for these amplitudes can be found in Ref. [7].

The cross sections for these processes with three particles in the final state can be expressed in terms of the off-shell cross sections of the subprocesses described by the amplitudes $M_{1}, M_{2}$, and $M_{3}$. Following the method of Ref. [16] for the reaction $N N \rightarrow N \Lambda K$, the spin and isospin averaged differential cross sections for the first two processes in Fig. 1 can be written as

$$
\begin{aligned}
\frac{d \sigma_{J / \psi N \rightarrow N D * \bar{D}}}{d t d s_{1}}= & \frac{g_{\pi N N}^{2}}{16 \pi^{2} s p_{i}^{2}} k \sqrt{s_{1}}(-t) \frac{F_{\pi N N}^{2}(t)}{\left(t-m_{\pi}^{2}\right)^{2}} \\
& \times \sigma_{J / \psi \pi \rightarrow D * \bar{D}}\left(s_{1}, t\right) \\
\frac{d \sigma_{J / \psi N \rightarrow N D \bar{D}}}{d t d s_{1}}= & \frac{3 g_{\rho N N}^{2}}{32 \pi^{2} s p_{i}^{2}} k \sqrt{s_{1}} \frac{F_{\rho N N}^{2}(t)}{\left(t-m_{\rho}^{2}\right)^{2}}\left[4\left(1+\kappa_{\rho}\right)^{2}\right. \\
& \times\left(-t-2 m_{N}^{2}\right) \kappa_{\rho}^{2} \frac{\left(4 m_{N}^{2}-t\right)^{2}}{2 m_{N}^{2}}+4\left(1+\kappa_{\rho}\right) \\
& \left.\times \kappa_{\rho}\left(4 m_{N}^{2}-t\right)\right] \sigma_{J / \psi \rho \rightarrow D \bar{D}}\left(s_{1}, t\right)
\end{aligned}
$$

and the differential cross section for $J / \psi N \rightarrow D^{*} \bar{D}^{*} N$ is similar to that for $J / \psi N \rightarrow D \bar{D} N$ with $\sigma_{J / \psi \rho \rightarrow D \bar{D}}\left(s_{1}, t\right)$ replaced by $\sigma_{J / \psi \rho \rightarrow D *} * \bar{D}^{*}\left(s_{1}, t\right)$.

In the above, $p_{i}$ is the center-of-mass momentum of $J / \psi$ and $N, t$ is the squared four momentum transfer, and $s_{1}$ and $k$ are, respectively, the squared invariant mass and center-ofmass momentum of $\pi$ and $J / \psi$ in the process $J / \psi N$ $\rightarrow D^{*} \bar{D} N$ or $\rho$ and $J / \psi$ in the processes $J / \psi N \rightarrow D \bar{D} N$ and 
$J / \psi N \rightarrow D^{*} \bar{D}^{*} N$. We have also introduced form factors $F_{\pi N N}$ and $F_{\rho N N}$ at the $\pi N N$ and $\rho N N$ vertices, respectively. As in Ref. [16], both are taken to have the monopole form, i.e.,

$$
F_{1}(t)=\frac{\Lambda^{2}-m^{2}}{\Lambda^{2}-t}
$$

where $m$ is the mass of exchanged pion or $\rho$ meson, and $\Lambda$ is a cutoff parameter. Following Refs. $[13,14]$, we take $\Lambda_{\pi N N}$ $=1.3 \mathrm{GeV}$ and $\Lambda_{\rho N N}=1.4 \mathrm{GeV}$.

The cross sections $\sigma_{J / \psi \pi \rightarrow D * \bar{D}}\left(s_{1}, t\right), \sigma_{J / \psi \rho \rightarrow D \bar{D}}\left(s_{1}, t\right)$, and $\sigma_{J / \psi \rho \rightarrow D *} \bar{D}^{*}\left(s_{1}, t\right)$ are the spin and isospin averaged differential cross sections for the subprocesses $J / \psi \pi$ $\rightarrow D^{*} \bar{D}, J / \psi \rho \rightarrow D \bar{D}$, and $J / \Psi \rho \rightarrow D^{*} \bar{D}^{*}$ with off-shell pion or $\rho$ meson. Explicit expressions for these cross sections can be obtained from Ref. [7] by replacing the square of pion or $\rho$ meson masses by $t$. In evaluating these cross sections, we also introduce form factors at the interaction vertices. Following Ref. [7], the form factors at three-point $t$ channel and $u$ channel vertices, i.e., $\pi D D^{*}, \rho D D, \rho D^{*} D^{*}, \psi D D$, and $\psi D^{*} D^{*}$ that involve heavy virtual charm mesons, are taken to have the following form:

$$
F_{2}\left(\mathbf{q}^{2}\right)=\frac{\Lambda^{2}}{\Lambda^{2}+\mathbf{q}^{2}},
$$

instead of the monopole form of Eq. (16). In the above, $\mathbf{q}$ is the three momentum transfer in the center of mass of $\psi$ and pion or $\rho$ meson.

The form factor at four-point vertices, i.e., $\pi \psi D D^{*}, \rho \psi D D$, and $\rho \psi D^{*} D^{*}$, are taken to be

$$
f_{4}=\left(\frac{\Lambda_{1}^{2}}{\Lambda_{1}^{2}+\left\langle\mathbf{q}^{2}\right\rangle}\right)\left(\frac{\Lambda_{2}^{2}}{\Lambda_{2}^{2}+\left\langle\mathbf{q}^{2}\right\rangle}\right),
$$

where $\Lambda_{1}$ and $\Lambda_{2}$ are the two different cutoff parameters at the three-point vertices present in processes with the same initial and final particles, and $\left\langle\mathbf{q}^{2}\right\rangle$ is the average value of the squared three momentum transfers in $t$ and $u$ channels.

Using the same value of $1 \mathrm{GeV}$ for cutoff parameters in the form factors involving charm mesons as in Refs. [7,8], we have evaluated the cross sections for $J / \psi$ absorption by nucleons, and they are shown in Fig. 2 as functions of total center-of-mass energy. It is seen that all cross sections are less than $2 \mathrm{mb}$. Furthermore, the cross section for $J / \psi N$ $\rightarrow D^{*} \bar{D} N$ or $J / \psi N \rightarrow \bar{D}^{*} D N$ (solid curve) due to pion exchange is larger than those for $J / \psi N \rightarrow D \bar{D} N$ (dashed curve) and $J / \psi N \rightarrow D^{*} \bar{D}^{*} N$ (dotted curve) that are due to $\rho$ meson exchange.

Our result for $\sigma_{J / \psi N \rightarrow D \bar{D} N}$ is order of magnitude smaller than that of Ref. [17], where this process is viewed as the elastic scattering of a nucleon with one of the charm mesons from the decay of $J / \psi$. The latter cross section is then assumed to have a constant value of $20 \mathrm{mb}$. Compared to our approach, they have neglected both the energy dependence

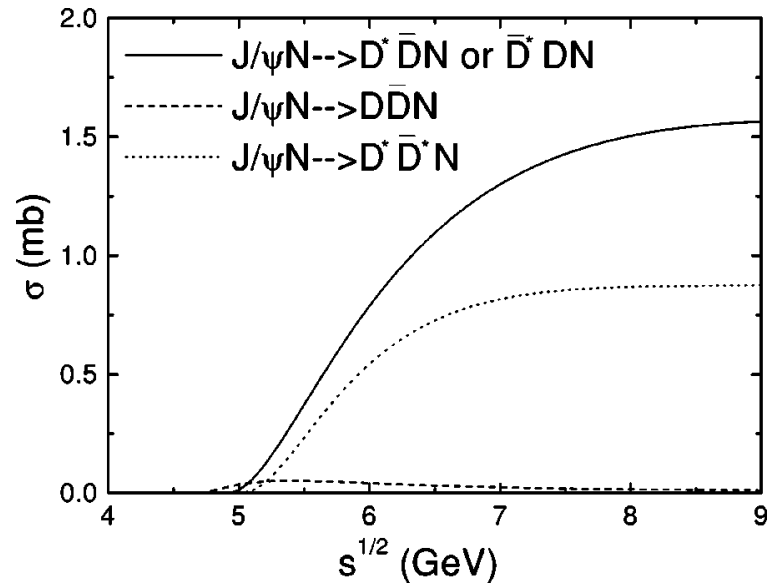

FIG. 2. Cross sections for $J / \psi$ absorption by nucleons due to the virtual pion and $\rho$ meson from the nucleon as functions of centerof-mass energy.

and the off-shell effect of the subprocess involved in $J / \psi-N$ absorption to three-body final state. Also contributing to this large difference in the cross section is the value of cutoff parameter, 3.1 GeV in Ref. [17] versus $1 \mathrm{GeV}$ used here, and the different momentum dependence, four momentum transfer in Ref. [17] while three momentum transfer in the present study. We note that the more important processes $J / \Psi N$ $\rightarrow D^{*} \bar{D} N\left(\bar{D}^{*} D N\right)$ and $J / \Psi N \rightarrow D^{*} \bar{D}^{*} N$ are not considered in Ref. [17].

\section{III. $J / \psi$ ABSORPTION BY NUCLEONS VIA CHARM EXCHANGE}

Besides absorption by the virtual pion and $\rho$ meson from nucleons, $J / \psi$ can also be absorbed by nucleons via charm exchange in the reaction $J / \psi N \rightarrow \bar{D} \Lambda_{c}$ and $J / \psi N \rightarrow \bar{D}^{*} \Lambda_{c}$ shown by the diagrams in Fig. 3. These processes involve the following interaction Lagrangians:
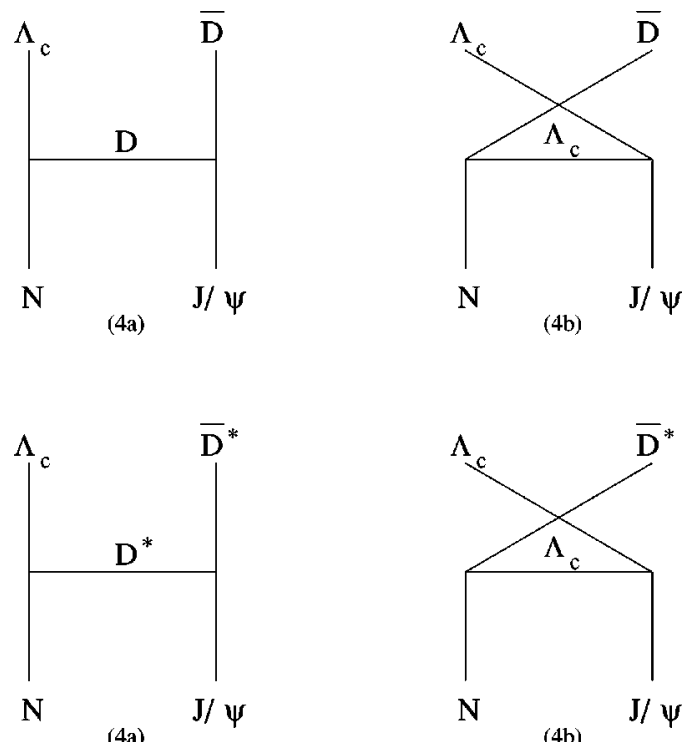

FIG. 3. $J / \psi$ absorption by nucleons via charm exchange. 


$$
\begin{gathered}
\mathcal{L}_{D N \Lambda_{c}}=i g_{D N \Lambda_{c}}\left(\bar{N} \gamma_{5} \Lambda_{c} \bar{D}+D \bar{\Lambda}_{c} \gamma_{5} N\right), \\
\mathcal{L}_{D^{* N \Lambda_{c}}}=g_{D^{* N \Lambda_{c}}}\left(\bar{N} \gamma_{\mu} \Lambda_{c} \bar{D}^{* \mu}+D^{* \mu} \bar{\Lambda}_{c} \gamma_{\mu} N\right), \\
\mathcal{L}_{\psi \Lambda_{c} \Lambda_{c}}=g_{\psi \Lambda_{c} \Lambda_{c}} \bar{\Lambda}_{c} \gamma^{\mu} \psi_{\mu} \Lambda_{c}
\end{gathered}
$$

where $\Lambda_{c}$ denotes the charm baryon.

The amplitudes for these processes are given by

$$
\begin{gathered}
M_{4 a}=M_{4 a}^{\mu} \varepsilon_{2 \mu}, \\
M_{4 b}=M_{4 b}^{\mu} \varepsilon_{2 \mu}, \\
M_{5 a}=M_{5 a}^{\mu \nu} \varepsilon_{2 \mu} \varepsilon_{4 \nu}, \\
M_{5 b}=M_{5 b}^{\mu \nu} \varepsilon_{2 \mu} \varepsilon_{4 \nu},
\end{gathered}
$$

with $\varepsilon_{2 \mu}$ and $\varepsilon_{4 \mu}$ being the polarization vectors of $J / \psi$ and $D^{*}$, respectively, and

$$
\begin{aligned}
& M_{4 a}^{\mu}=2 i g_{\psi D D} g_{D N \Lambda_{c}} \frac{1}{t-m_{D}^{2}} p_{4}^{\mu} \bar{\Lambda}_{c}\left(p_{3}\right) \gamma_{5} N\left(p_{1}\right), \\
& M_{4 b}^{\mu}=i g_{D N \Lambda_{c}} g_{\psi \Lambda_{c} \Lambda_{c}} \bar{\Lambda}_{c}\left(p_{3}\right) \gamma^{\mu} \frac{q+m_{\Lambda_{c}}}{u-m_{\Lambda_{c}}^{2}} \gamma_{5} N\left(p_{1}\right), \\
& M_{5 a}^{\mu \nu}=-g_{D^{*} N \Lambda_{c}} g_{\psi D^{*} D^{*}} \bar{\Lambda}_{c}\left(p_{3}\right) \gamma^{\alpha} N\left(p_{1}\right) \\
& \times\left[g_{\alpha \beta}-\frac{\left(p_{1}-p_{3}\right)_{\alpha}\left(p_{1}-p_{3}\right)_{\beta}}{m_{D^{*}}^{2}}\right] \frac{1}{t-m_{D^{*}}^{2}} \\
& \times\left[p_{2}^{\nu} g^{\beta \mu}-\left(p_{2}+p_{4}\right)^{\beta} g^{\mu \nu}+2 p_{4}^{\mu} g^{\beta \nu}\right], \\
& M_{5 b}^{\mu \nu}=g_{D^{* N \Lambda_{c}}} g_{\psi \Lambda_{c} \Lambda_{c}} \bar{\Lambda}_{c}\left(p_{3}\right) \gamma^{\mu} \frac{q+m_{\Lambda_{c}}}{u-m_{\Lambda_{c}}^{2}} \gamma^{\nu} N\left(p_{1}\right) .
\end{aligned}
$$

In the above, $q=p_{1}-p_{4}, s=\left(p_{1}+p_{2}\right)^{2}$, and $t=\left(p_{1}-p_{3}\right)^{2}$ are the standard Mendelstam variables.

The spin and isospin averaged differential cross sections for these two-body processes are then

$$
\begin{aligned}
& \frac{d \sigma_{\psi N \rightarrow \bar{D} \Lambda_{c}}}{d t}=\frac{1}{64 \pi s p_{i}^{2}}\left|M_{4 a}+M_{4 b}\right|^{2}, \\
& \frac{d \sigma_{\psi N \rightarrow \bar{D} * \Lambda_{c}}}{d t}=\frac{1}{64 \pi s p_{i}^{2}}\left|M_{5 a}+M_{5 b}\right|^{2},
\end{aligned}
$$

where $\left|M_{4 a}+M_{4 b}\right|^{2}$ and $\left|M_{5 a}+M_{5 b}\right|^{2}$ can be evaluated using the software package FORM [18].

The coupling constants $g_{D N \Lambda_{c}}, g_{D *_{N \Lambda_{c}}}$, and $g_{\psi \Lambda_{c} \Lambda_{c}}$ can be related to known coupling constants $g_{\pi N N}$ and $g_{\rho N N}$ using the $\mathrm{SU}(4)$ symmetry as shown in the Appendix. Using $g_{\pi N N}=13.5$ and $g_{\rho N N}=3.25$, we then have $g_{D N \Lambda_{c}}$ $=13.5, g_{D^{*} N \Lambda_{c}}=-5.6$, and $g_{\psi \Lambda_{c} \Lambda_{c}}=-1.4$. We again intro-

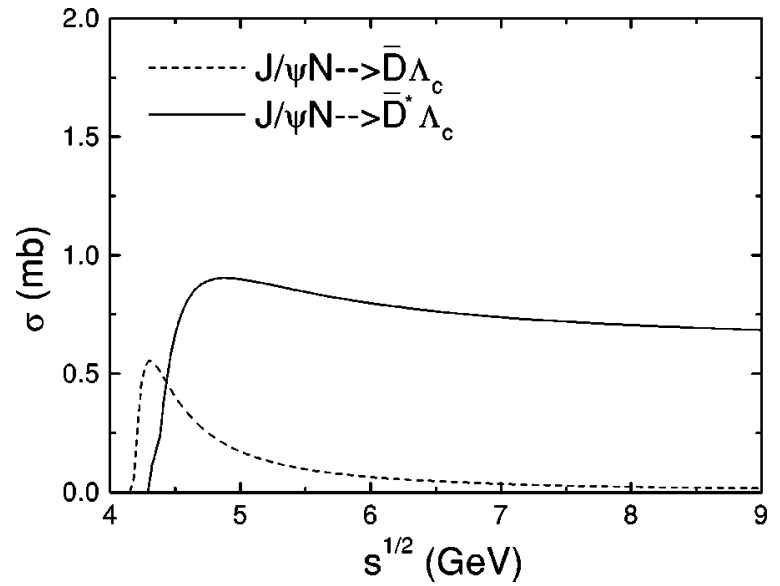

FIG. 4. Cross sections for $J / \psi$ absorption by nucleons due to charm exchange as functions of center-of-mass energy.

duce monopole form factors of Eq. (17) at the vertices with cutoff parameter $\Lambda=1 \mathrm{GeV}$. The resulting cross sections for $J / \psi N \rightarrow \bar{D} \Lambda_{c}$ and $J / \psi N \rightarrow \bar{D}^{*} \Lambda_{c}$ are shown in Fig. 4 by the dashed and solid curves, respectively. Their values are seen to be less than $1 \mathrm{mb}$. Furthermore, $\sigma_{J / \psi N \rightarrow \bar{D} * \Lambda_{c}}$ is much larger than $\sigma_{J / \psi N \rightarrow \bar{D} \Lambda_{c}}$ due to the three-vector-mesons coupling, which has been shown to increase significantly the $J / \psi-\pi$ absorption cross section as well [7].

In Ref. [17], only diagram (4a) in Fig. 3 has been studied, and the result there is about a factor of 4 larger than our cross section for $J / \psi N \rightarrow \bar{D} \Lambda_{c}$, which includes also diagram (4b). The larger cross section in Ref. [17] is again due to both a larger cutoff parameter of $2 \mathrm{GeV}$ versus $1 \mathrm{GeV}$ used here and the use of four momentum instead of three momentum transfer in the form factors. Our total $J / \psi-N$ absorption cross section due to charm exchange is, however, larger as we have also included the more important processes shown by Figs. (5a) and (5b).

\section{THE ANOMALOUS PARITY INTERACTION}

There is also anomalous parity interaction of $J / \psi$ with charm mesons [8], i.e.,

$$
\mathcal{L}_{\psi D * D}=g_{\psi D^{* D}} \varepsilon_{\alpha \beta \mu \nu}\left(\partial^{\alpha} \psi^{\beta}\right)\left[\left(\partial^{\mu} \bar{D}^{* \nu}\right) D+\bar{D}\left(\partial^{\mu} D^{* \nu}\right)\right],
$$

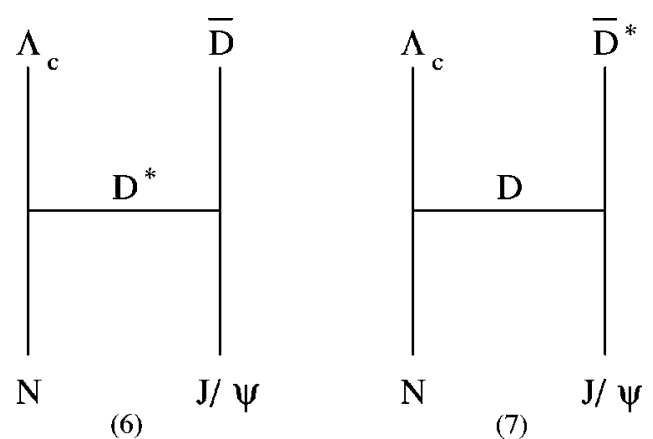

FIG. 5. $J / \psi$ absorption by nucleons via charm meson exchange through the anomalous parity interaction. 
which not only introduces additional diagrams for the processes shown in Fig. 1 but also leads to the reactions $J / \psi N$ $\rightarrow \bar{D} \Lambda_{c}$ via $D^{*}$ exchange and $J / \psi N \rightarrow \bar{D}^{*} \Lambda_{c}$ via $D$ exchange shown by the diagrams in Fig. 5.

As shown in Ref. [8], the anomalous parity interaction is not important for $J / \psi-\rho$ absorption and increases the $J / \psi-\pi$ absorption cross section by only about $50 \%$. Thus, inclusions of additional diagrams due to the anomalous parity interaction in processes involving three-body final states shown in Fig. 1 will probably increase the $J / \psi-N$ absorption cross section calculated here by less than $50 \%$.

The amplitudes for the process $J / \psi N \rightarrow \bar{D} \Lambda_{c}$ and $J / \psi N$ $\rightarrow \bar{D}^{*} \Lambda_{c}$ due to the anomalous parity interaction are given by

$$
\begin{gathered}
M_{6}=M_{6}^{\mu} \varepsilon_{2 \mu}, \\
M_{7}=M_{7}^{\mu \nu} \varepsilon_{2 \mu} \varepsilon_{4 \nu},
\end{gathered}
$$

with $\varepsilon_{2 \mu}$ and $\varepsilon_{4 \nu}$ being the polarization vectors of $J / \psi$ and $D^{*}$, respectively, and

$$
\begin{aligned}
& M_{6}^{\mu}=-g_{\psi D^{*} D} g_{D^{*} N \Lambda_{c}} \frac{1}{t-m_{D^{*}}^{2}} \varepsilon^{\mu \nu \alpha \beta} p_{2 \alpha}\left(p_{1}-p_{3}\right)_{\beta} \\
& \times \bar{\Lambda}_{c}\left(p_{3}\right) \gamma_{\nu} N\left(p_{1}\right) \\
& M_{7}^{\mu \nu}= i g_{\psi D^{*} D} g_{D N \Lambda_{c}} \frac{1}{t-m_{D}^{2}} \varepsilon^{\mu \nu \alpha \beta} p_{2 \alpha} p_{4 \beta} \\
& \times \bar{\Lambda}_{c}\left(p_{3}\right) \gamma_{5} N\left(p_{1}\right) .
\end{aligned}
$$

Because of the anomalous parity in the $\psi D^{*} D$ vertex, the process $J / \psi N \rightarrow \bar{D} \Lambda_{c}$ via $D^{*}$ exchange does not interfere with the similar process via $D$ exchange shown in Fig. 3 . The differential cross sections for the two anomalous processes in Fig. 5 are given by similar expressions as Eqs. (29) and (30) with

$$
\begin{aligned}
\left|M_{6}\right|^{2}= & \frac{g_{\psi D * D}^{2} g_{D * N \Lambda_{c}}^{2}}{12 m_{\psi}^{2}} \frac{1}{\left(t-m_{D^{*}}^{2}\right)^{2}}\left\{4 m _ { \psi } ^ { 2 } \left[2\left(m_{N}^{2}+m_{\Lambda_{c}}^{2}\right) t-t^{2}\right.\right. \\
& \left.-\left(m_{\Lambda_{c}}^{2}-m_{N}^{2}\right)^{2}\right]+2\left(m_{\Lambda_{c}}^{2}-m_{N}^{2}\right)\left[\left(m_{\psi}^{2}+m_{\Lambda_{c}}^{2}-u\right)^{2}\right. \\
& \left.-\left(s-m_{N}^{2}-m_{\psi}^{2}\right)^{2}\right]-\left[2\left(m_{N}^{2}+m_{\Lambda_{c}}^{2}\right)-t\right]\left(2 m_{\psi}^{2}+m_{\Lambda_{c}}^{2}\right. \\
& \left.+m_{N}^{2}-u-s\right)^{2}-t\left(m_{\Lambda_{c}}^{2}-m_{N}^{2}+s-u\right)^{2} \\
& -2\left[\left(m_{N}-m_{\Lambda_{c}}\right)^{2}-t\right]\left[4 m_{\psi}^{2} t-\left(2 m_{\psi}^{2}+m_{N}^{2}+m_{\Lambda_{c}}^{2}\right.\right. \\
& \left.\left.-u-s)^{2}\right]\right\},
\end{aligned}
$$

and

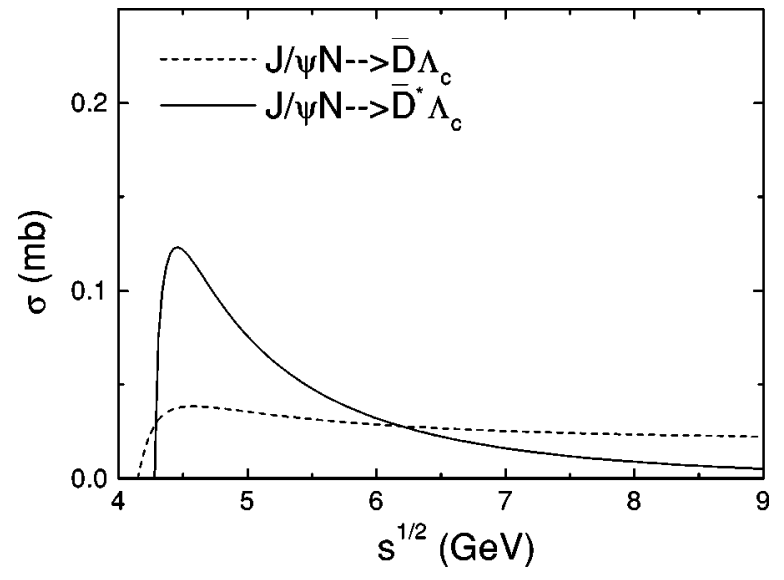

FIG. 6. Cross sections for $J / \psi$ by nucleons as functions of center-of-mass energy due to the anomalous parity interaction.

$$
\begin{aligned}
\left|M_{7}\right|^{2}= & \frac{g_{\psi D * D}^{2} g_{D N \Lambda_{c}}^{2}}{6 m_{\psi}^{2}} \frac{1}{\left(t-m_{D}^{2}\right)^{2}}\left[\left(m_{N}-m_{\Lambda_{c}}\right)^{2}-t\right] \\
& \times\left[\left(m_{\psi}^{2}+M_{D^{*}}^{2}-t\right)^{2}-m_{\psi}^{2} m_{D^{*}}^{2}\right],
\end{aligned}
$$

where $u=\left(p_{1}-p_{4}\right)^{2}$.

The coupling constant in the anomalous parity interaction has been determined to be $g_{\psi D D^{*}}=8.61 \mathrm{GeV}^{-1}$ from the radiative decay of $D^{*}$ to $D$ using the vector dominance model [8]. With a monopole form factor similar to Eq. (17) at the $D^{*} N \Lambda_{c}$ vertex and a cutoff parameter of $1 \mathrm{GeV}$, the cross sections for the reactions $J / \psi N \rightarrow D \Lambda_{c}$ due to $D^{*}$ exchange and $J / \psi N \rightarrow D^{*} \Lambda_{c}$ due to $D$ exchange are shown in Fig. 6. Their values are seen to be less than $0.15 \mathrm{mb}$, which is negligible compared to the contributions from normal interactions studied in Sec. II and III.

We note that the two processes in Fig. 5 due to the anomalous parity interaction have also been studied in Ref. [17]. Their coupling constant is related to ours by $g_{\psi D D^{*}} / m_{J / \psi}$, where $m_{J / \psi}$ is the mass of $J / \psi$. Since they assume that $g_{\psi D D^{*}}=g_{\psi D D}=7.64$ based on an incorrect quotation from Ref. [19], the strength of the anomalous coupling constant in their study is only $2.47 \mathrm{GeV}^{-1}$ and is about a factor of 3 smaller than that used here. However, they have used a much larger value for $g_{D * N \Lambda_{c}}=-19$ than that given by the SU(4) relation. As a result, their cross section for diagram (7) in Fig. 5 should have a similar magnitude as ours while that of diagram (6) should be larger than our value. Because of the larger value of cutoff parameter of $2 \mathrm{GeV}$ and the use of four momentum transfer in the form factor, the results in Ref. [17] from the anomalous parity interaction turn out to be order of magnitude larger than ours.

\section{TOTAL CROSS SECTION FOR $J / \psi$ ABSORPTION BY NUCLEONS}

The total cross section for $J / \psi$ absorption by nucleons, obtained by adding the contributions shown in Figs. 2, 4, and 6 is given in Fig. 7. At low center-of-mass energies, the cross section is dominated by the process $J / \psi N \rightarrow \bar{D}^{*} \Lambda_{c}$ while at 


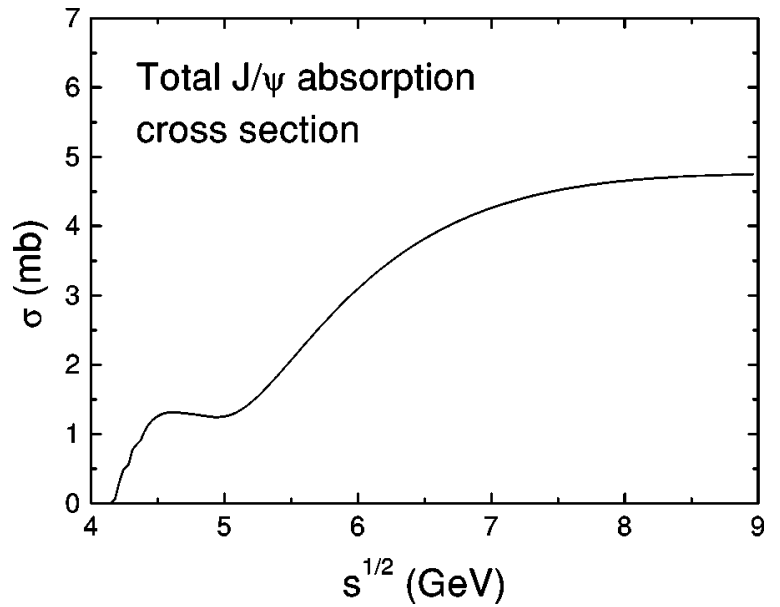

FIG. 7. Total $J / \psi$ absorption cross sections by nucleon as a function of center-of-mass energy.

high center-of-mass energies, the processes $J / \psi N \rightarrow D^{*} \bar{D} N$ and $J / \psi N \rightarrow \bar{D}^{*} D N$ due to the virtual pion from the nucleon are most important. The total $J / \psi$ absorption cross section is at most $5 \mathrm{mb}$ and is consistent with that extracted from $J / \psi$ production in photonucleus and proton-nucleus reactions.

We note that the total cross section we obtained for $J / \psi$ absorption by nucleons turns out comparable to that in Ref. [17], although contributions from individual processes differ appreciably in the two studies as discussed in details in the end of preceding sections.

\section{CONCLUSIONS AND DISCUSSIONS}

We have used a meson-exchange model to study the cross section for $J / \psi$ absorption by nucleons. The interaction Lagrangians are based on the gauged SU(4) flavor symmetry but with empirical masses. Using coupling constants taken either from the empirical information or via the SU(4) relations, and form factors with cutoff parameter of $1 \mathrm{GeV}$, we obtain a $J / \psi$-nucleon absorption cross section of at most 5 $\mathrm{mb}$, which is consistent with the empirical cross section extracted from $J / \psi$ production in photonucleus and protonnucleus reactions. Since the dominant process can be viewed as $J / \psi$ absorption by the virtual pion from the nucleon, our results thus indicate that the cross sections for $J / \psi$ absorption by pion and $\rho$ meson evaluated in previous studies using the meson-exchange model are not in contradiction with the empirical cross section for $J / \psi$ absorption by nucleons.

Our results are not much affected if we use the coupling constants $g_{D N \Lambda_{c}} \sim 6.7-7.9$ and $g_{D^{*} N \Lambda_{c}} \sim-7.5$ determined from the QCD sum rules [20] instead from the SU(4) symmetry. With these values, $\sigma_{\psi N \rightarrow \bar{D} \Lambda_{c}}$ will be even smaller while $\sigma_{\psi N \rightarrow \bar{D} * \Lambda_{c}}$ will be about a factor of 2 larger than those shown in Fig. 4. In this case, the $J / \psi-N$ absorption cross section is only increased by about $1 \mathrm{mb}$. On the other hand, if the cutoff parameter is taken to be $\Lambda=2 \mathrm{GeV}$ at vertices involving charm hadrons as suggested by QCD sum rules [20], then the total $J / \psi-N$ absorption cross section increases to about $10 \mathrm{mb}$, which is about a factor of 2 larger than the empirical value from $J / \psi$ production in photonucleus and proton-nucleus reactions. With this cutoff parameter, the $J / \psi-\pi$ absorption cross section is also about $10 \mathrm{mb}$ as shown in Ref. [7]. Since the meson-exchange model is based on effective hadronic Lagrangians, one can either fit the empirical $J / \psi-N$ absorption cross section by treating the cutoff parameter as a phenomenological parameter, or use the cutoff parameter from the QCD sum rules but with a different effective Lagrangian. In the former case, a cutoff parameter of $1 \mathrm{GeV}$ is required at the interaction vertices involving charm hadrons in order to have the correct $J / \psi-N$ absorption cross section. The meson-exchange model of Ref. [7] then gives a $J / \psi-\pi$ absorption cross section of about $3 \mathrm{mb}$, which is also consistent with that used in the comover model for $J / \psi$ suppression in heavy ion collisions $[3,21]$. In the latter case, one may follow the suggestion of Ref. [22] to drop the nongradient pion couplings in the effective Lagrangians, as they break the chiral $\mathrm{SU}(2) \times \mathrm{SU}(2)$ symmetry. As shown in Ref. [22], neglecting these terms reduces the $J / \psi-\pi$ absorption cross section by about a factor of 2 , leading again to a $J / \psi-\pi$ absorption cross section similar to that in the comover model. The $J / \psi-N$ absorption cross section obtained with such an effective Lagrangian is expected to be reduced as well.

\section{ACKNOWLEDGMENTS}

We thank M. Nielsen and A. Sibirtsev for their comments on the manuscript. This work was supported by the National Science Foundation under Grant No. PHY-9870038, the Welch Foundation under Grant No. A-1358, and the Texas Advanced Research Program under Grant No. FY99010366-0081.

\section{APPENDIX}

In the SU(4) quark model, baryons belong to the 20-plet states. These states can be conveniently expressed by tensors $\phi_{\mu \nu \lambda}$ [23], where $\mu, \nu$, and $\lambda$ run from 1 to 4 , that satisfy the conditions

$$
\phi_{\mu \nu \lambda}+\phi_{\nu \lambda \mu}+\phi_{\lambda \mu \nu}=0, \quad \phi_{\mu \nu \lambda}=\phi_{\nu \mu \lambda} .
$$

For baryons without charm quarks, i.e., belonging to $\mathrm{SU}(3)$ octet, they are given by

$$
\begin{gathered}
p=\phi_{112}, \quad n=\phi_{221}, \quad \Lambda=\sqrt{\frac{2}{3}}\left(\phi_{321}-\phi_{312}\right), \\
\Sigma^{+}=\phi_{113}, \quad \Sigma^{0}=\sqrt{2} \phi_{123} \quad \Sigma^{-}=\phi_{223}, \\
\Xi^{0}=\phi_{331}, \quad \Xi^{-}=\phi_{332} .
\end{gathered}
$$

For baryons with one charm quark, they are

$$
\Sigma_{c}^{++}=\phi_{114}, \quad \Sigma_{c}^{+}=\phi_{124}, \quad \Sigma_{c}^{0}=\phi_{224},
$$




$$
\begin{gathered}
\Xi_{c}^{+}=\phi_{134}, \quad \Xi_{c}^{0}=\phi_{234}, \\
\Xi_{c}^{+^{\prime}}=\sqrt{\frac{2}{3}}\left(\phi_{413}-\phi_{431}\right), \quad \Xi_{c}^{0^{\prime}}=\sqrt{\frac{2}{3}}\left(\phi_{423}-\phi_{432}\right), \\
\Lambda_{c}^{+}=\sqrt{\frac{2}{3}}\left(\phi_{421}-\phi_{412}\right), \quad \Omega_{c}^{0}=\phi_{334} .
\end{gathered}
$$

For baryons with two charm quarks, they are

$$
\Xi_{c c}^{++}=\phi_{441}, \quad \Xi_{c c}^{+}=\phi_{442}, \quad \Omega_{c c}^{+}=\phi_{443} .
$$

Mesons in the SU(4) quark model belong to the 15 plet. In the tensor notations, pseudoscalar and vector mesons are expressed by $P_{\beta}^{\alpha}$ and $V_{\beta}^{\alpha}$, respectively. For pseudoscalar mesons, we have

$$
\begin{gathered}
\pi^{+}=P_{1}^{2}, \quad \pi^{-}=P_{2}^{1}, \quad \pi^{0}=\frac{1}{\sqrt{2}}\left(P_{1}^{1}-P_{2}^{2}\right), \\
K^{+}=P_{1}^{3}, \quad K^{0}=P_{2}^{3}, \quad K^{-}=P_{3}^{1}, \quad \bar{K}^{0}=P_{3}^{2}, \\
D^{+}=P_{4}^{2}, \quad D^{0}=P_{4}^{1}, \quad D^{-}=P_{2}^{4}, \quad \bar{D}^{0}=P_{1}^{4}, \\
D_{s}^{+}=P_{4}^{3}, \quad D_{s}^{-}=P_{3}^{4}, \\
\eta=\frac{1}{\sqrt{6}}\left(P_{1}^{1}+P_{2}^{2}-2 P_{3}^{3}\right), \\
\eta_{c}=\frac{1}{\sqrt{12}}\left(P_{1}^{1}+P_{2}^{2}+P_{3}^{3}-3 P_{4}^{4}\right) .
\end{gathered}
$$

Similarly, we have for vector mesons

$$
\begin{gathered}
\rho^{+}=V_{1}^{2}, \quad \rho^{-}=V_{2}^{1}, \quad \rho^{0}=\frac{1}{\sqrt{2}}\left(V_{1}^{1}-V_{2}^{2}\right), \\
K^{*+}=V_{1}^{3}, \quad K^{* 0}=V_{2}^{3}, \quad K^{*-}=V_{3}^{1}, \quad \bar{K}^{* 0}=V_{3}^{2}, \\
D^{*+}=V_{4}^{2}, \quad D^{* 0}=V_{4}^{1}, \quad D^{*-}=V_{2}^{4}, \quad \bar{D}^{* 0}=V_{1}^{4}, \\
D_{s}^{*+}=V_{4}^{3}, \quad D_{s}^{*-}=V_{3}^{4}, \\
\omega=\frac{1}{\sqrt{6}}\left(V_{1}^{1}+V_{2}^{2}-2 V_{3}^{3}\right), \\
J / \psi=\frac{1}{\sqrt{12}}\left(V_{1}^{1}+V_{2}^{2}+V_{3}^{3}-3 V_{4}^{4}\right) .
\end{gathered}
$$

In tensor notations, the $\mathrm{SU}(4)$ invariant interaction Lagrangians between baryons and pseudoscalar mesons as well as between baryons and vector mesons can be written, respectively, as

$$
\begin{gathered}
\mathcal{L}_{P B B}=g_{p}\left(a \phi^{* \alpha \mu \nu} \gamma_{5} P_{\alpha}^{\beta} \phi_{\beta \mu \nu}+b \psi^{* \alpha \mu \nu} \gamma_{5} P_{\alpha}^{\beta} \phi_{\beta \nu \mu}\right), \\
\mathcal{L}_{V B B}=g_{v}\left(c \phi^{* \alpha \mu \nu} \gamma V_{\alpha}^{\beta} \phi_{\beta \mu \nu}+d \phi^{* \alpha \mu \nu} \gamma V_{\alpha}^{\beta} \phi_{\beta \nu \mu}\right),
\end{gathered}
$$

where $g_{p}$ and $g_{v}$ are universal baryon-pseudoscalar-meson and baryon-vector-meson coupling constants, and $a, b, c$, and $d$ are constants.

Writing explicitly, we obtain the following interaction Lagrangians,

$$
\begin{aligned}
\mathcal{L}_{P B B}= & g_{p}\left[\frac{1}{\sqrt{2}}\left(a-\frac{5}{4} b\right) \bar{N} \gamma_{5} \vec{\tau} \cdot \vec{\pi} N+\frac{3 \sqrt{6}}{8}(b-a)\left(\bar{N} \gamma_{5} K \Lambda\right.\right. \\
& \left.\left.+\bar{N} \gamma_{5} \bar{D} \Lambda_{c}\right)+\cdots\right], \\
\mathcal{L}_{V B B}= & g_{v}\left[\frac{1}{\sqrt{2}}\left(c-\frac{5}{4} d\right) \bar{N} \gamma_{\mu} \rho^{\mu} N+\frac{3 \sqrt{6}}{8}(d-c)\left(\bar{N} \gamma_{\mu} K^{* \mu} \Lambda\right.\right. \\
& \left.+\bar{N} \gamma_{\mu} \bar{D}^{\mu *} \Lambda_{c}\right)+\frac{\sqrt{3}}{4}\left(-c+\frac{3}{2} d\right) \bar{\Lambda}_{c} \gamma_{\mu} \psi^{\mu} \Lambda_{c} \\
& +\cdots] .
\end{aligned}
$$

The baryon-pseudoscalar-meson coupling in $\mathrm{SU}(3)$ is usually written as $\operatorname{DTr}[(B \bar{B}+\bar{B} B) M]+F \operatorname{Tr}[(B \bar{B}-\bar{B} B) M]$, where $B$ and $M$ are the baryon and pseudoscalar meson octets. In terms of the ratio $\alpha_{D}=D /(D+F)$, which has an empirical value of about 0.64 [24], we then have the following relation between $g_{\pi N N}$ and $g_{K N \Lambda}$ in the Lagrangians $\mathcal{L}_{\pi N N}$ given by Eq. (1) and $\mathcal{L}_{K N \Lambda}=i g_{K N \Lambda} \bar{N} \gamma_{5} \Lambda \bar{K}$,

$$
g_{K N \Lambda}=\frac{3-2 \alpha_{D}}{\sqrt{3}} g_{\pi N N},
$$

Comparisons with the SU(4) relations in Eq. (A9) then gives

$$
\frac{b}{a}=\frac{3-8 \alpha_{D}}{6-10 \alpha_{D}}
$$

The baryon-vector-meson coupling is usually introduced through the minimal coupling by treating vector mesons as gauge particles. In $\mathrm{SU}(3)$, this leads to the following relation between $g_{\rho N N}$ and $g_{K^{* N \Lambda}}$ in the Lagrangians $\mathcal{L}_{\rho N N}$ given by Eq. (2) and $\mathcal{L}_{K^{*} N \Lambda}=g_{K^{*} N \Lambda} \bar{N} \gamma_{\mu} \Lambda \bar{K}^{*}$, 


$$
g_{K * N \Lambda}=-\sqrt{3} g_{\rho N N} .
$$

Comparing with the $\mathrm{SU}(4)$ relations in Eq. (A10), we have

$$
\frac{d}{c}=\frac{1}{2}
$$

Using Eqs. (A12) and (A14) in Eqs. (A9) and (A10), we then have

$$
g_{D N \Lambda_{c}}=\frac{3-2 \alpha_{D}}{\sqrt{3}} g_{\pi N N},
$$

$$
g_{\psi \Lambda_{c} \Lambda_{c}}=-\frac{g_{\rho N N}}{\sqrt{6}}, \quad g_{D * N \Lambda c}=-\sqrt{3} g_{\rho N N},
$$

for the coupling constants in the Lagrangians given by Eqs. (19), (20), and (21).
[1] M. Gonin et al., NA50 Collaboration, Phys. Lett. B 450, 456 (1999).

[2] T. Matsui and H. Satz, Phys. Lett. B 178, 416 (1986).

[3] J. Ftacnik, P. Lichard, and J. Pisut, Phys. Lett. B 207, 194 (1988); S. Gavin, M. Gyulassy, and A. Jackson, ibid. 207, 257 (1988); R. Vogt, M. Prakash, P. Koch, and T.H. Hansson, ibid. 207, 263 (1988); S. Gavin Nucl. Phys. A566, 287c (1994); R. Vogt, Phys. Rep. 310, 197 (1999).

[4] D. Kharzeev and H. Satz, Phys. Lett. B 334, 155 (1994).

[5] K. Martins, D. Blaschke, and E. Quack, Phys. Rev. C 51, 2723 (1995); C.Y. Wong, E.S. Swanson, and T. Barnes, ibid. 62, 045201 (2000).

[6] K.L. Haglin, Phys. Rev. C 61, 031902(R) (2000).

[7] Z.W. Lin and C.M. Ko, Phys. Rev. C 62, 034903 (2000).

[8] Y. Oh, T. Song, and S.H. Lee, Phys. Rev. C 63, 034901 (2000).

[9] F.S. Navarra, M. Nielsen, R.S. Marques de Carvalho, and G. Krein, nucl-th/0105058.

[10] R.L. Anderson et al., Phys. Rev. Lett. 38, 263 (1977).

[11] D. Kharzeev, C. Lourenco, M. Nardi, and H. Satz, Z. Phys. C 74, 307 (1997).

[12] Z.W. Lin, T.G. Di, and C.M. Ko, Nucl. Phys. A689, 965 (2001).

[13] B. Holzenkamp, K. Holinde, and J. Speth, Nucl. Phys. A500,
485 (1989); G. Janssen, J.W. Durso, K. Holinde, B.C. Pearce, and J. Speth, Phys. Rev. Lett. 71, 1978 (1993).

[14] G. Janssen, K. Holinde, and J. Speth, Phys. Rev. C 54, 2218 (1996).

[15] S.G. Matinyan and B. Müller, Phys. Rev. C 58, 2994 (1998).

[16] T. Yao, Phys. Rev. 125, 1048 (1961).

[17] A. Sibirtsev, K. Tsushima, and A.W. Thomas, Phys. Rev. C 63, 044906 (2001).

[18] J. Vermaseren, computer code FORM, 1989. Free version of the software is available on the Internet at ftp:// hep.itp.tuwien.ac.at/pub/Form/PC/

[19] Z.W. Lin, C.M. Ko, and B. Zhang, Phys. Rev. C 61, 024904 (2000).

[20] F.S. Navarra and M. Nielsen, Phys. Lett. B 443, 285 (1998); F.O. Durães, F.S. Navarra, and M. Nielsen, ibid. 498, 169 (2001).

[21] W. Cassing and C.M. Ko, Phys. Lett. B 396, 39 (1997); W. Cassing and E.L. Bratkovkaya, Nucl. Phys. A623, 570 (1997).

[22] F.S. Navarra, M. Nielsen, and M.R. Robilotta, Phys. Rev. C 64, 021901(R) (2001).

[23] S. Okubo, Phys. Rev. D 11, 3261 (1975).

[24] R.A. Adelseck and B. Saghai, Phys. Rev. C 42, 108 (1990). 